\title{
Sistema hipermedia adaptativo para el apoyo del aprendizaje autónomo del idioma inglés
}

\author{
Eugenia Erica Vera ${ }^{1}$, Yadira Navarro ${ }^{2}$ Josefina Guerrero $^{1}$ \\ ${ }^{1}$ Facultad de Ciencias de la Computación, \\ Benemérita Universidad Autónoma de Puebla, Puebla, México \\ ${ }^{2}$ Facultad de Ciencias de la Electrónica, \\ Benemérita Universidad Autónoma de Puebla, Puebla, México \\ \{eevclibra, ynavarro44, joseguga01\}@gmail.com
}

\begin{abstract}
Resumen. Hoy en día es más evidente la diversidad de los alumnos en el aprendizaje de inglés, encontramos estudiantes con facilidad de comprensión tanto en lectura como en gramática compartiendo el aula con estudiantes que requieren más tiempo para alcanzar un desenvolvimiento adecuado en el manejo del idioma. El potencial de las TIC puede favorecer en diversas formas los procesos de autoaprendizaje y posibilitar distintas modalidades educativas para apoyar la enseñanza aprendizaje del idioma. El propósito de este artículo es presentar el diseño y desarrollo de un Sistema Hipermedia Adaptativo para el apoyo del aprendizaje autónomo del idioma inglés y en particular promover la comprensión auditiva y oral del mismo. El sistema considera las características del estudiante (historial), para adaptar y presentar los contenidos educativos. También se presentan los resultados obtenidos del sistema al realizar una prueba piloto con una muestra de estudiantes.
\end{abstract}

Palabras clave: Sistema Hipermedia Adaptativo, multimedia, aprendizaje de inglés.

\section{Adaptive Hypermedia System for the Support of Auton- omous Learning of the English Language}

\begin{abstract}
Presently the diversity of the students in the learning of English is becoming more evident, we found students with easy comprehension, both in reading and in grammar, sharing the classroom with students that require more time to reach an adequate development in the use of the language. The potential of ICTs can favor self-learning processes in different ways and enable different educational modalities to support the teaching and learning of the language. The purpose of this article is to present the design and development of an Adaptive Hypermedia System for the support of the autonomous learning of the English language, particularly to promote the auditory and oral comprehension of it. The
\end{abstract}




\begin{abstract}
system considers the characteristics of the student (record), to adapt and present the educational contents. The results obtained from the system when conducting a pilot test with a sample of students are also presented.
\end{abstract}

Keywords. Adaptive Hypermedia System, multimedia, learning English.

\title{
1. Introducción
}

A la mayoría de las personas se les dificulta entablar y mantener una conversación en el idioma inglés, a pesar de que es una de las materias que han cursado en su preparación básica. Entre las principales causas se tienen, el tiempo que los alumnos dedican al estudio y practica del idioma, la estructura de los planes curriculares que se basan en enseñar la gramática en forma tradicional, lo que ocasiona que los alumnos abandonen el curso una y otra vez. Por lo anterior es importante desarrollar nuevas herramientas que faciliten el aprendizaje de este idioma.

Este proyecto se centra en desarrollar una herramienta que estimula la comprensión auditiva y la comunicación del idioma inglés, tomando en cuenta que actualmente la generación de estudiantes tiene acceso a numerosa información que se encuentra en internet.

El objetivo es estimular y desarrollar el oído fonemático, para mejorar la comprensión auditiva a través de actividades audiovisuales, para lo cual se desarrollaron actividades multimedia con las que el alumno trabaja de manera autónoma y éstas se apegan a sus necesidades y disponibilidad, logrando facilitar el aprendizaje.

Los estudios revelan que el estilo docente no se relaciona de manera significativa con el rendimiento académico de los alumnos, es por ello que trabajamos en apoyar al estudiante en el contexto del aprendizaje autónomo como proceso individual que depende del trabajo, de los hábitos de estudio y de las estrategias del alumno.

El documento está conformado y organizado de la siguiente manera: en la sección 2, se presenta la fundamentación teórica del Marco Común Europeo, acerca de la competencia lingüística, la explicación del concepto de un Sistema Hipermedia Adaptativo (SHA), la conceptualización de aprendizaje autónomo, así como los temas y; subtemas a considerar y competencias lingüísticas a desarrollar; en la sección 3, se describe la metodología de la investigación; en la sección 4, se presenta el desarrollo y pruebas del SHA y, finalmente en la sección 5, se exponen las conclusiones y trabajos a futuro.

\section{Fundamentación teórica}

\subsection{Marco común europeo de referencia para las lenguas}

El Marco Común Europeo de Referencia para las Lenguas es, principalmente, el estándar internacional que define la competencia lingüística. Se utiliza para la elaboración de programas de lenguas, orientaciones curriculares, exámenes, manuales, etc., en todo el mundo. En él se describe lo que tienen que aprender a hacer los estudiantes de 
lenguas con el fin de utilizar una lengua para comunicarse, así como los conocimientos y destrezas que tienen que desarrollar para poder actuar de manera eficaz.

El enfoque que ha adoptado el Marco Común se centra en la acción y en la medida en que considera a los usuarios y alumnos que aprenden una lengua principalmente como agentes sociales, es decir, como miembros de una sociedad que tienen tareas (no sólo relacionadas con la lengua) que llevar a cabo en una serie determinada de circunstancias, en un entorno específico y dentro de un campo de acción concreto.

El marco común señala seis niveles que cubren adecuadamente el proceso de aprendizaje de una lengua. Los niveles son: 1) Acceso (Breakthrough), 2) Plataforma (Waystage), 3) Umbral (Threshold), 4) Avanzado (Vantage), 5) Dominio operativo eficaz (Effective Operational Proficiency) y 6) Maestría (Mastery).

Por lo que los tres grados comunes: Básico, Intermedio y Avanzado ó A, B, y C sitúan a los seis niveles como sigue:

Básico (A): A1: Acceso (Breakthrough), A2: Plataforma (Waystage).

Intermedio (B): B1:Umbral (Threshold), B2: Avanzado (Vantage).

Avanzado (C): C1: Dominio operativo eficaz y C2: Maestría (Mastery).

\subsection{Sistema Hipermedia Adaptativo (SHA)}

Brusilovsky define un SHA como un sistema basado en hipertexto e hipermedia que refleja algunas características del usuario y aplica un modelo para adecuar varios aspectos del sistema al usuario [4]. Aquellos sistemas de hipermedia capaces de ajustar su presentación y navegación a los diferentes usuarios reducen los problemas de desorientación y falta de comprensión de estos mismos usuarios (propios de los sistemas hipermedia no adaptativos) [5]. Un sistema se considera adaptativo cuando se ajusta de forma automática y personalizada a las necesidades del usuario [4]. Por lo que un SHA permite personalizar la información almacenada y la presenta a los usuarios según sus preferencias, conocimientos e intereses. El proceso de personalización muestra la información que es apropiada para algún tipo de conocimiento y de aprendizaje de cada usuario. El modelo posee un conjunto de reglas que permiten adaptar los contenidos al perfil del usuario, considerando sus características y el tipo de contenido que debe aprender para cumplir con los objetivos, logrando manifestar relaciones interesantes a partir de la información existente.

Los SHA Educativos son sistemas que, basados en el grado de conocimiento del usuario construyen rutas de aprendizaje adaptativas, personalizando la adquisición de conocimiento y adaptando el material que es recomendado.

Según P. Brusilovsky, un SHA debe cumplir los siguientes criterios [4]:

1. Ser un sistema hipertexto o hipermedia,

2. Tener un modelo de usuario

3. Ser capaz de adaptar el hipermedia usando este modelo.

Uno de los objetivos más relevantes para el cual un SHA es desarrollado, es que el sistema se adapte al usuario y no sea el usuario quien deba adaptarse al sistema, como sucede regularmente en los hipermedia "clásicos", los cuales muestran el mismo contenido y los mismos enlaces a todos los usuarios. Las soluciones con la hipermedia 
adaptativa son: mejor comprensión de la información (rutas de navegación seleccionadas), y orientación en el hiperespacio. Es importante considerar el nivel de conocimiento y ritmo de aprendizaje del usuario.

En el SHA que se presenta en este trabajo considera el perfil académico con el que cuenta el estudiante, considerando el nivel de conocimientos y habilidades en el idioma inglés y canal preferente de aprendizaje.

\subsection{Método eclecticismo de enseñanza}

Desde una perspectiva ecléctica (The New Principled Eclecticism Method), que da la oportunidad a los docentes de utilizar y tomar metodologías y enfoques, así como seleccionar técnicas de cada método que consideren efectivas para aplicarlas de acuerdo al contexto y los objetivos aprendizaje [15], en el SHA se consideran dos diferentes métodos o estrategias de enseñanza; el auditivo lingual y el método basado en tareas.

El método Audio lingual sugiere que a los estudiantes se les debe de enseñar el idioma de manera directa, es decir sin utilizar la lengua materna del estudiante para explicar nuevas palabras o gramática del idioma que están aprendiendo [16].

Con base en las necesidades del SHA, este método es muy útil porque las habilidades se enseñan en el siguiente orden: escuchar, hablar, leer y, escribir. Considerando que, la habilidad auditiva es importante para desarrollar la competencia del habla y de esta manera, recibe un énfasis en particular.

De acuerdo con Brown las características principales de este método son [8]:

- La enseñanza se presenta por medio del diálogo.

- Hay poca o nula explicación gramatical.

- La gramática enseña de manera inductiva.

- El vocabulario se aprende en contexto.

- Se utilizan audios y material visual.

- Se le da prioridad a la pronunciación.

El método basado en tareas (Task-based method), es el método que ve el proceso de aprendizaje como un set de tareas de comunicación que están directamente conectadas con una meta curricular y propósitos que se extienden más allá de la práctica del idioma; esto significa que los alumnos usan el lenguaje ya aprendido para completar tareas o actividades [17].

La mayoría de los métodos que se conocen en el área de la enseñanza del inglés funcionan con las cuatro habilidades principales y cada una de ellas depende de la otra para conseguir sus objetivos, por lo que no es común que se enseñe una sola habilidad, es decir aislada de las demás; por ejemplo, hablar y escuchar se necesitan para poder comprender con exactitud una conversación, y en algunos contextos, leer o escuchar y tomar notas son parte una conversación. Las actividades de integración de habilidades en el SHA incluyen, por ejemplo, escuchar audios, aprender con juegos, o trabajar con paquetes de información y ejercicios para resolver problemas; este tipo de práctica requieren que los estudiantes se involucren en el idioma y que además se involucren en la integración. 


\subsection{Aprendizaje autónomo}

Una característica importante del SHA es el aprendizaje autónomo que se considera como un aprendizaje estratégico en el que la persona toma decisiones claves sobre su propio aprendizaje: autodirigiéndolo en función de unas necesidades, metas o propósitos, auto regulándolo (seleccionando alternativas, acciones, tiempos) y autoevaluándolo, de acuerdo con los recursos y escenarios de que dispone y de las exigencias y condiciones del contexto. Con el aprendizaje autónomo la persona aprende a aprender gracias al entrenamiento y desarrollo de competencias o habilidades cognitivas, afectivas e interactivas, pero también, y de manera esencial, gracias al desarrollo de habilidades metacognitivas [10].

Tabla 1. Temas y subtemas para el SHA.

\begin{tabular}{ll}
\hline Tema & Subtemas \\
\hline 1. Simple Present & 1.1. Introduce yourself \\
& 1.2. Greetings \\
& 1.3. Habits and routines \\
2. Present Continuous & 2.1. What are you doing? \\
3. Past Simple & 3.1. What did you do last week? \\
& 3.2. Regular and irregular verbs \\
& 3.3. Time Expressions \\
4. Past Continuous & 4.1. How I met my best friend \\
& 4.2. My childhood \\
5. Future: Will / Shall & 5.1. What will you do when you graduate? \\
6. Future: Going to & 6.1. What are you going to do on your vacations? \\
7. Modal Verbs & 7.1. Can: abilities \\
& 7.2. Could: possibilities \\
8. Perfect present & 7.3. Should: advices \\
& 8.1. Extreme sports and food \\
9. Wh Questions & 8.2. Have you ever...? \\
10. Nouns & 9.1. News \\
\hline
\end{tabular}

\subsection{Temas y subtemas para el SHA}

El contenido temático que se consideró en el SHA considera las funciones que el alumno debe realizar con el idioma a un nivel A2, conforme al Marco Común Europeo (ver tabla 1). Como objetivo general de aprendizaje se establece que el alumno será capaz de comunicar necesidades simples y básicas, así como desarrollar funciones sociales tales como: saludar, presentarse, preguntar como están y conseguir información sencilla, manejando los tres tiempos: presente, pasado y futuro.

\subsection{Competencias}

Las competencias a desarrollar mediante el SHA para la comunicación en el idioma inglés se presentan en la tabla 2 . 
Tabla 2. Competencias a desarrollar para la comunicación en el idioma inglés.

\begin{tabular}{ll}
\hline Competencias & \multicolumn{1}{c}{ Atributos } \\
\hline & $\begin{array}{l}\text { El alumno será capaz de presentarse, así como presentar a alguien más, sabrá reconocer distintas } \\
\text { maneras de saludar y hablará sobre sus hábitos y rutinas. }\end{array}$ \\
El alumno será capaz de describir las actividades que otras personas están realizando. \\
2. Se expresa y & El alumno será capaz de narrar las actividades que realizó la semana pasada, y reconocerá los \\
comunica & verbos regulares e irregulares. \\
& El alumno será capaz de narrar un momento específico de su vida. \\
& El alumno expresará sus planes a futuro. \\
& El alumno será capaz de describir lo que hará en sus vacaciones. \\
& El alumno identifica los diferentes tempos de las oraciones. \\
3. Piensa crítica y & El alumno identificara las diferencias entre los verbos modales, así como las situaciones en las \\
reflexivamente & El alumno será capaz de preguntar y responder sobre actividades inusuales. \\
& El alumno será capaz de describir alguna noticia relevante que haya escuchado. \\
4.Aprende de & El alumno desarrollara habilidades auditivas y orales. \\
forma autónoma & El alumno será capaz de seleccionar la técnica para comprender y producir información en \\
5.Trabaja en & idioma inglés. \\
forma colabora- & El alumno será capaz de comunicar necesidades simples y básicas, así como desarrollar funcio- \\
tiva & nes sociales en forma colaborativa. \\
\hline
\end{tabular}

\section{Metodología}

El SHA se diseñó y desarrolló dentro de la metodología de innovación aplicada, propuesta por el grupo CIDER (creatividad e innovación para el desarrollo regional) de la Universidad Politécnica de Valencia (UPV) [2], por las ventajas que representaba el permitir generar referentes para buscar nuevas estrategias para la mejora. Esta metodología interpreta y combina las tendencias del mercado, la tecnología disponible y los movimientos sociales para resolver las necesidades, en nuestro caso la enseñanza del inglés como lengua extranjera y lograr superar las expectativas de los usuarios [3].

En este estudio se consideró una muestra de 308 adultos de 15 a 55 años, 123 niños y 308 adolescentes de 12 a 14 años; del instituto DMSV de capacitación para el trabajo, en el primer trimestre de otoño 2016.

En el paso de la Abstracción, el estudio consistió en analizar el servicio y se concluye que el instituto ofrece una gran variedad de cursos en inglés, y los costos están al alcance de la mayoría de las personas, el $78.64 \%$ de alumnos adultos que ingresan al instituto, se ubican en el nivel Básico; el $18.93 \%$ en el nivel intermedio y sólo el $2.43 \%$ en un nivel avanzado. En el grupo de niños el $72 \%$ se ubican en el nivel Básico, el $28 \%$ en el nivel intermedio y ninguno en avanzado. En el grupo de adolescentes el $87 \%$ que ingresa al instituto se ubican en el nivel Básico, el $13 \%$ en el nivel intermedio y ninguno en avanzado. Estos niveles se ajustan de acuerdo al Marco Común Europeo. Este paso permitió definir el contenido temático para el cual desarrollamos el SHA.

En el paso de Conocimiento que es el que pretende monitorizar a los actores, nuestro estudio se dirige al grupo de los adultos, para el cual es el producto a desarrollar. Mediante un cuestionario pretendemos detectar las necesidades de estos actores. El cuestionario está formado de nueve preguntas, con respuesta de opción múltiple documentado en [3]. De los resultados obtenidos, se consideraron en el SHA, los siguientes: el $64 \%$ de la muestra considera que la prioridad es lograr mantener una conversación, un $30 \%$ considera que es el poder redactar textos en inglés, y un $4 \%$ requieren de entender 
las lecturas en este idioma, el menor porcentaje $2 \%$ y como última prioridad requieren de certificaciones como del MARCO COMÚN EUROPEO o el TOFEL. El $80 \%$ de los estudiantes desea dominar la lengua en menos de un año. Los estudiantes revelan que no tienen tiempo de estudiar el idioma y la dedicación al estudio de este es de 2 horas cada tercer día o ningún momento, son pocos los libros de textos que leen y si los leen es porque se los dejaron de tarea y la mayoría de las veces son artículos relaciones con sus respectivas carreras, ven videos y películas de vez en cuando. Lo que cabe resaltar es que la mayoría prefiere material didáctico en línea por que pueden acceder.

En el paso de análisis de tendencias intersectorial: Existen institutos que ofrecen cursos que cubren sin problema las necesidades marcadas por los alumnos encuestados, omitimos nombres por cuestiones de derechos de autor, sin embargo estos cursos, comúnmente se pagan con anticipación a un precio no muy accesible para el público en general.

Los curso online están ganado terreno, sin embargo se requieren competencias tecnológicas previas de los estudiantes, comúnmente las tareas y objetivos de aprendizaje, de estos cursos, no están bien descritos y es fácil que los estudiantes dejen los estudios a medias o no aprovechen al máximo la formación.

En el paso de definición de escenarios se tiene que una de las prioridades de la población es mantener una conversación, ya que piensan que en la conversación pone en práctica la gramática y pueden comprobar si realmente entiendes la conversación de la otra persona que está hablando, es por esto que se debe seleccionar, de los planes de estudio, las partes que realmente aportan al aprendizaje y desarrollo de la habilidad de habla y de escucha del idioma. Requieren lograr estos objetivos en menos de un año, es por ello que se necesita priorizar y jerarquizar los aprendizajes, programar las actividades e incluir en estas varias competencias para lograr en menos tiempo los objetivos.

Solo le dedican una hora a estudiar, esto nos lleva a crear actividades que motiven al estudiante, enseñarle a utilizar todo lo que le rodea para aprender. La mayoría prefiere material didáctico en línea por que pueden acceder a la hora que tengan disponible, por lo que debemos programar los materiales y actividades en línea. Los alumnos cuentan con diferentes conocimientos por lo que es importante reforzar los nuevos conocimientos y saltarse los ya aprendidos, en esta parte el SHA adapta las actividades según el perfil del estudiante.

\section{Desarrollo y pruebas del Sistema Hipermedia Adaptativo para el apoyo del aprendizaje autónomo del idioma inglés}

El sistema se desarrolló bajo el modelo cliente-servidor donde todo comienza con una petición o requerimiento HTTP iniciado en un navegador por un cliente que quiere acceder a un recurso de nuestro sitio web por medio de una dirección URL. La dirección URL apunta a la localización física de una página de extensión .ASP. 


\subsection{Actividades de aprendizaje autónomo}

Las actividades de aprendizaje autónomo son acciones específicas que facilitan la ejecución de la estrategia de aprendizaje autónomo y el desarrollo de competencias. Estas actividades hacen énfasis en el proceso intelectual que se encuentra especificado para cada idea principal y además son la base del diagnóstico académico así que las preguntas se presentan de la manera más clara posible, para que el alumno no caiga en confusiones. En cada actividad se le presenta al alumno la información del tema y subtema que está cursando, involucren los niveles cognoscitivos, para lo cual se tomaron como referencia los cinco niveles cognoscitivos que describe Bloom (tabla 3).

Es importante disponer de información suficiente para resaltar los aprendizajes relevantes de cada unidad, es decir diferenciar un aprendizaje importante de uno secundario.

Para definir cuáles son los aprendizajes relevantes de las unidades, temas o áreas que se seleccionaron para ser diagnosticadas, se tiene el siguiente método que consta de tres pasos [12]:

a) Seleccionar las ideas principales o esenciales de cada una de las unidades, temas o áreas elegidas.

b) Determinar los procesos cognoscitivos deseables para cada idea esencial. Una vez que se seleccionaron las ideas principales del contenido de la enseñanza, se realiza un análisis de cada una de ellas para determinar los procesos intelectuales que el alumno pondrá en juego al haberlas aprendido.

Los procesos intelectuales característicos del aprendizaje escolar utilizados son [12]: Conocimiento (repetir y reconocer información), comprensión (ejemplificar, comparar e interpretar la idea esencial), aplicación (integrar varias ideas, aplicar la idea esencial en situaciones nuevas o en la solución del problema), análisis (analizar la idea principal), síntesis y evaluación cognitivo.

Al final obtendremos una puntuación de las ideas principales (conceptos, hechos, procedimientos), junto con el señalamiento de los procesos cognitivos que se esperan para cada uno de ellos.

c) Señalar el tipo de aprendizaje referido en cada idea esencial, en función al programa de estudios. El aprendizaje se clasifica en diferentes formas. Quesada propone hacerlo en tres categorías: indispensable, esencial y antecedente [12].

Los aprendizajes relevantes conforman parte del perfil académico deseado del alumno. Una vez que se tiene definido por completo el perfil académico deseado (aprendizajes relevantes) del alumno, así como sus antecedentes, se comienza la elaboración del instrumento diagnóstico, mediante el cual se le evaluará.

Se diseñaron cinco tipos de actividades: Booleana, Opción Múltiple, Completar Texto, Relacionar y Arrastrar. En cada actividad es posible que el alumno se encuentre con un poco de indecisión al momento de responder a su actividad, en este caso todas las actividades cuentan con la opción de regresar a la clase que observaron antes de dicha actividad, como ayuda para resolver dudas, sin otorgar directamente la respuesta. 
Tabla 3. Atributos de las actividades de aprendizaje autónomo.

\begin{tabular}{|c|c|c|c|c|c|c|c|c|c|c|c|c|c|c|c|c|}
\hline \multicolumn{3}{|c|}{ Actividad } & \multicolumn{3}{|c|}{ Competencia } & \multicolumn{3}{|c|}{$\begin{array}{c}\text { Tipo de aprendi- } \\
\text { zaje } \\
\text { (Quesada,1996) }\end{array}$} & \multicolumn{6}{|c|}{$\begin{array}{l}\text { Proceso cognitivo (Bloom, 2004) } \\
\text { (perfil deseable) }\end{array}$} & \multicolumn{2}{|c|}{$\begin{array}{l}\text { Perfil ac- } \\
\text { tual del } \\
\text { estu- } \\
\text { diante }\end{array}$} \\
\hline$\stackrel{-1}{\stackrel{9}{\Xi}}$ & $\frac{\mathscr{\mathscr { V }}}{\tilde{\Xi}}$ & 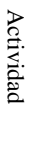 & 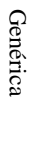 & 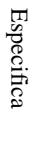 & $\begin{array}{l}\text { D. } \\
\text { 咅 } \\
\text { ठ }\end{array}$ & 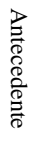 & 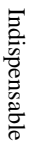 & $\begin{array}{l}\text { TI } \\
0 \\
0 \\
\stackrel{0}{0} .\end{array}$ & 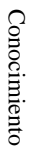 & 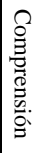 & 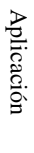 & 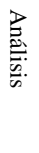 & 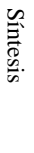 & 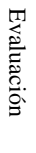 & 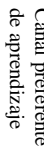 & 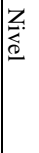 \\
\hline 1. & 1.1 & 1 & 4 & 7 & 28 & $\mathrm{X}$ & & & & & $\mathrm{X}$ & & & & $\mathrm{A}$ & $\mathrm{B}$ \\
\hline 2. & 2.1 & 2 & 4 & 7 & 28 & $\mathrm{X}$ & & & & & $\mathrm{X}$ & & & & $\mathrm{A}$ & $\mathrm{M}$ \\
\hline 2. & 1.2 & 3 & 4 & 7 & 28 & & $\mathrm{X}$ & & $\mathrm{X}$ & & & & & & $\mathrm{V}$ & B \\
\hline 2. & 1.3 & 4 & 4 & 7 & 28 & & & & & & $\mathrm{x}$ & & & & $\mathrm{V}$ & A \\
\hline
\end{tabular}

Nota: $\mathrm{A}=$ Auditivo, $\mathrm{V}=$ Visual, $\mathrm{K}=$ Kinestésico, $\mathrm{B}=\mathrm{Bajo}, \mathrm{M}=$ medio, $\mathrm{A}=$ Alto

\subsection{Desarrollo del Sistema educativo}

En la Fig. 1 se observa que el usuario tiene acceso al Sistema educativo a través de links que le permitirán irse desplazando por todo el Sistema.

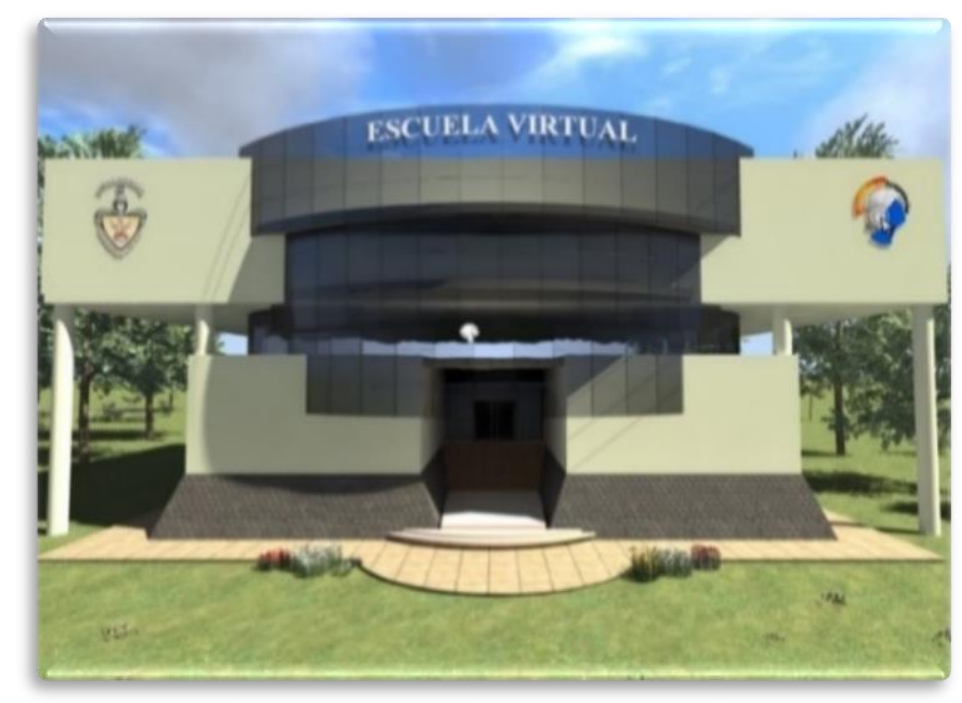

Fig. 1. Entrada al Sistema educativo para el aprendizaje del idioma inglés.

Una vez dentro de la escuela, se muestran vínculos, a los que se podrán acceder a: Aula, Cafetería, Biblioteca, Secretaria, Tablón de anuncios, Correo, Tareas Programadas, Ayuda y desplazarse por el sitio. En la sección Aula encontraremos foros, contenidos de la materia, ideas clave, herramientas multimedia, actividades de aprendizaje, un archivador, y tablón de anuncios (ver Fig. 2). 


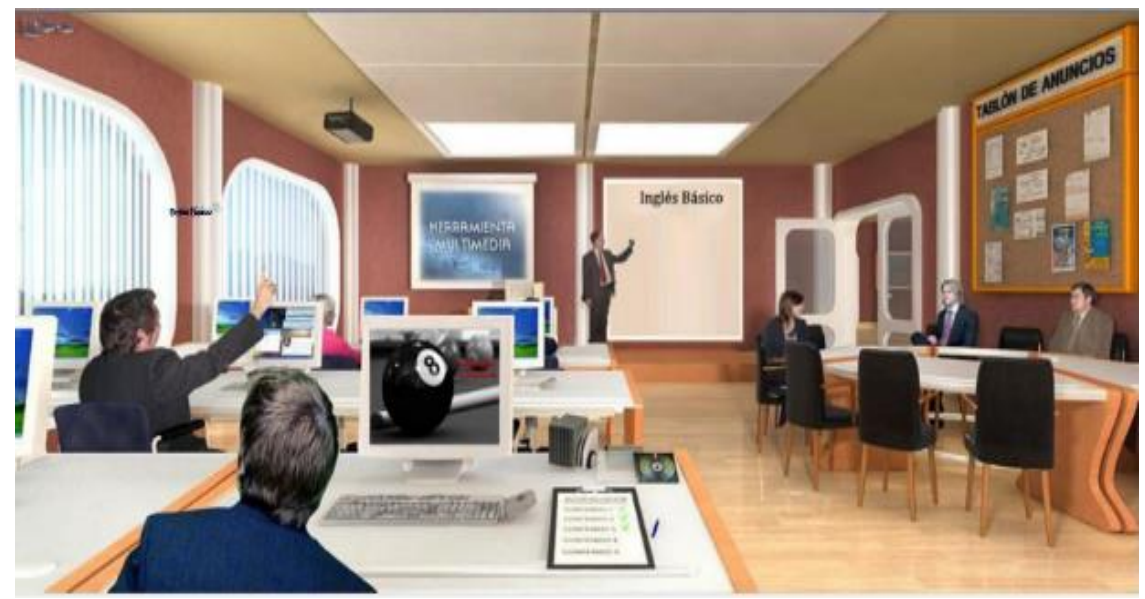

Fig. 2. Dentro del aula en el Sistema educativo.

Una vez que seleccionamos la materia y el tema, el sistema pide que se identifique el estudiante, mediante una clave de acceso.

El Sistema educativo tiene programadas las clases de todo el curso, permitiendo al alumno seleccionar la clase de aprendizaje que desea aprender o repasar. En la figura 3 se muestra una clase mediante un video.

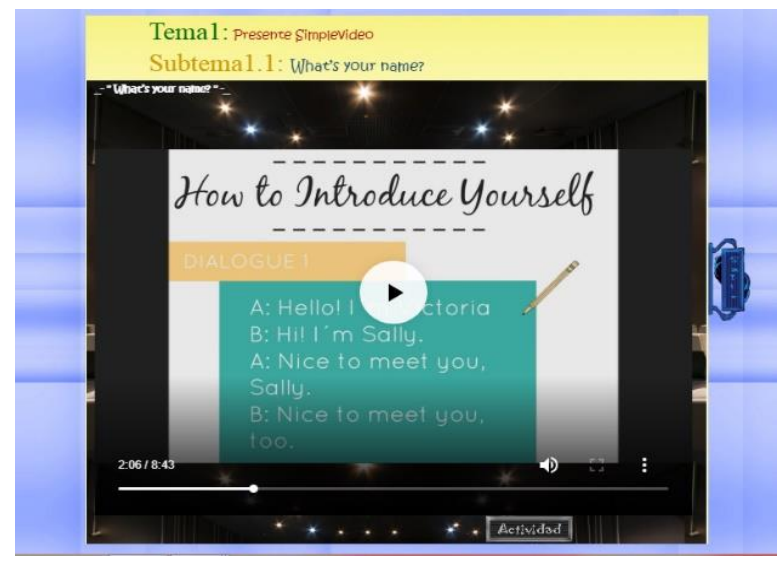

Fig. 3. Pantalla de una clase de aprendizaje de inglés básico en el Sistema educativo.

El Sistema educativo tiene programadas las actividades en ambientes virtuales para el aprendizaje, en donde los estudiantes reciban una instrucción y al mismo tiempo son partícipes de ella jugando un rol activo para fomentar la colaboración de los mismos y enriqueciendo el acervo que el sistema ofrezca para dicho aprendizaje. En la figura 4 se muestra un ejemplo de actividad de aprendizaje, de tipo opción múltiple. 


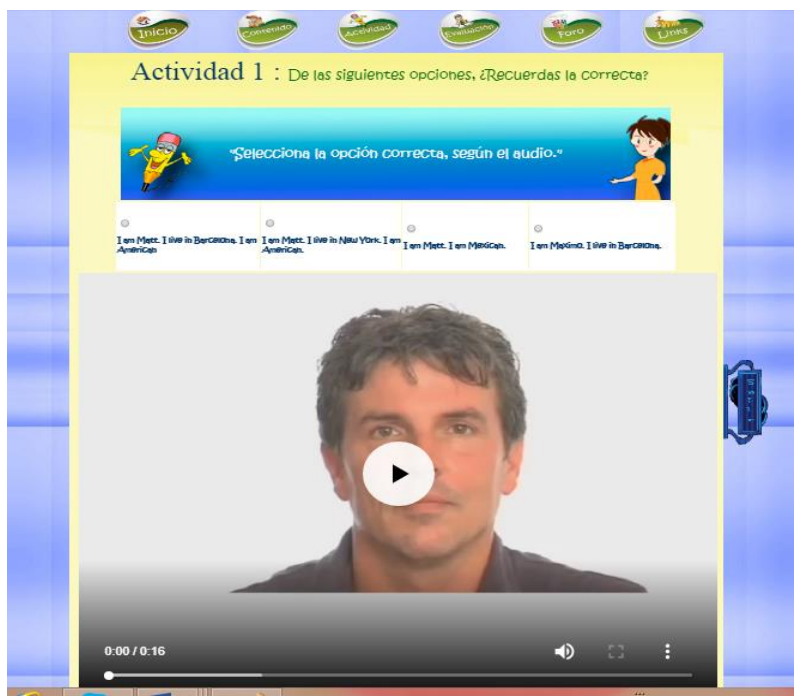

Fig. 4. Pantalla de una actividad de aprendizaje, de tipo opción múltiple, de inglés básico en el Sistema educativo.

En la Fig. 5 se observa una actividad de relacionar el concepto con la imagen. En caso de que el alumno falle en su respuesta, se determinó que no se le puede decir literalmente que no tiene validez, así que en los dos tipos de respuesta, correcta o no, se guiará con un tipo de apoyo para aclararle cual camino era el indicado, esto sin darles la respuesta, simplemente como una idea que despejará sus dudas y motivando a continuar con el curso. En el momento de contestar acertadamente el concepto, se le aumentará los puntos que le corresponden.

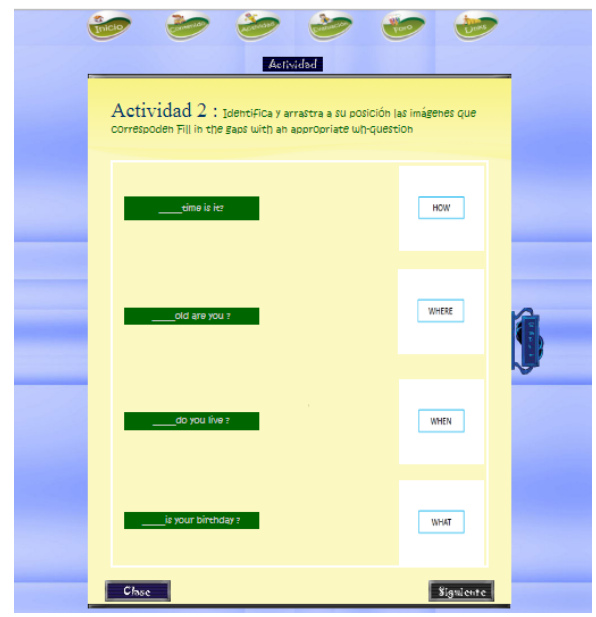

Fig. 5. Pantalla de una actividad de arrastrar el concepto con la imagen. 
La información de los movimientos del alumno es almacenada en una bitácora para consultas posteriores, es decir, se guarda un seguimiento del alumno y los movimientos realizados en sus actividades. Al finalizar las actividades, el sistema muestra el nivel con el que terminó y da la opción para borrar su historial. Este nivel está determinado por la cantidad de preguntas que maneja el sistema y los puntos adquiridos con sus respuestas correctas.

Están registrados en la base de datos los nombres de los todos académicos de dicha materia, se le asignó como password su matrícula única, y su primer apellido.

Las funciones que puede desempeñar un profesor en el Sistema educativo multimedia son las siguientes:

- Ver el contenido temático de la materia.

- Ver la lista de sus alumnos con información de avances.

En la elaboración de las preguntas o actividades a realizar por el alumno se considera el proceso intelectual que se toma en cuenta para cada una de ellas, y dependiendo del tipo de aprendizaje que cada una representa. Dentro de este proceso se le otorgó una ponderación a cada tipo de aprendizaje únicamente como ayuda para establecer el nivel en el que el alumno se encuentra, ya sea, alto, medio o bajo. La ponderación mencionada es: al conocimiento indispensable 6 puntos, al conocimiento esencial 4 puntos y al conocimiento antecedente 6 puntos.

\subsection{Resultados}

El SHA fue piloteado con alumnos adultos de los cursos básicos del instituto en estudio con una muestra $n=32$, cuyo objetivo fue obtener el diagnóstico inicial y final. Las evaluaciones permitieron revisar el nivel de logro del desarrollo de las competencias en comunicación y comprensión del idioma inglés que adquirieron los alumnos durante el ciclo escolar 2017, siendo el sistema una herramienta de apoyo utilizada con los alumnos de forma presencial durante las sesiones de clases.

A todos, es decir, a los 32 estudiantes se les aplicó la prueba protocolo, al inicio del curso para asegurar igualdad en cuanto a sus estrategias de aprendizaje apegado a las dimensiones manejadas en la Escala de Estrategias de Aprendizaje Contextualizado (ESEAC) (Bernad, 2007).

Las cuatro competencias que se midieron son: Comprender información auditiva en el idioma inglés, Comunicar información oral mediante el idioma inglés, Comprensión lectora del idioma inglés y Manejo de técnicas eficientes donde cada una representa un $25 \%$ del total de las competencias y a partir de esta información es como se realiza la evaluación.

Los estudiantes tomaron el curso a lo largo de 3 meses, con los mismos contenidos, y finalmente se evalúo nuevamente la prueba protocolo con el fin de observar el avance en las estrategias de aprendizaje. En la tabla 4 se presentan los resultados iniciales y finales del grupo control.

La competencia que más se desarrolló fue "Comprender información auditiva en el idioma inglés" con un $65 \%$ de desarrollo, de $2 \%$ a un $65 \%$ y la segunda fue "El manejo de técnicas eficientes" con un $60 \%$, logrando alcanzar de un $20 \%$ a un $60 \%$ y la tercera 
"Comunicar información oral mediante el idioma inglés", logrando alcanzar de un 5\% a un $40 \%$, esto conlleva que el uso de un SHA propicia un aprendizaje más significativo en el tema del idioma inglés, como se muestra en la Tabla 4.

Tabla 4. Resultados del Test Inicial, Test Final y aportación del desarrollo para el desarrollo de competencias mediante el SHA.

\begin{tabular}{lccc}
\hline \multicolumn{1}{c}{ Competencias } & $\begin{array}{c}\text { Test Ini- } \\
\text { cial }\end{array}$ & $\begin{array}{c}\text { Test } \\
\text { Final }\end{array}$ & $\begin{array}{c}\text { Uso } \\
\text { Sistema }\end{array}$ \\
\hline Comprender información auditiva en el idioma inglés & $2 \%$ & $65 \%$ & $63 \%$ \\
El manejo de técnicas eficientes & $20 \%$ & $60 \%$ & $40 \%$ \\
Comunicar información oral mediante el idioma in- & $5 \%$ & $40 \%$ & $35 \%$ \\
glés & & & \\
\hline
\end{tabular}

\section{Conclusiones}

Tomando en cuenta el enfoque de competencias en las nuevas teorías de aprendizaje y considerando los modelos de sistemas de cómputo se logró el diseño y desarrollo de un Sistema Adaptativo Hipermedia para el apoyo del aprendizaje autónomo de idioma inglés.

Se logró promover la comprensión auditiva y oral de este idioma gracias a la potencialidad que nos ofrece la tecnología multimedia.

Considerando las características del estudiante (historial), para adaptar y presentar los contenidos educativos en dicho sistema se lograron adecuar las actividades de aprendizaje convenientes para el desarrollo de las competencias en cuanto al manejo del idioma inglés.

Se logró aumentar la motivación de los estudiantes, y el desarrollo de la habilidad de comprensión auditiva del idioma inglés.

Se sistematizaron estrategias para el logro de un aprendizaje autónomo, y se aplicaron metodologías para atender la diversidad.

En cuanto a la comprensión de información auditiva de los estudiantes según la prueba protocolo, se logró un avance significativo al contrastar los resultados iniciales con los finales en el grupo experimental.

Se puede asegurar que el sistema puede ser utilizado de manera generalizada en todos los grupos de la materia de inglés como refuerzo para el aprendizaje, sin interferir con el estilo de enseñanza.

Se pretende, en un futuro, desarrollar las actividades de aprendizaje de todos los niveles del Marco Común Europeo.

\section{Referencias}

1. Antich de León, R.: Metodología de la enseñanza de las Lenguas Extranjeras. Editorial Pueblo y Educación, La Habana (1986)

2. Guijarro, J.: Desarrollo de una metodología para la gestión del proceso de innovación. una perspectiva aplicada, Valencia (2006) 
3. Vera, E., López, E.C., Guerrero, J.: Sistema didáctico e-Learning para el apoyo del aprendizaje del idioma inglés (2016)

4. Brusilovsky, P.: Methods and techniques of adaptive hypermedia. User Modeling and User Adapted Interaction. Netherlands, v 6, 2-3, 87-129. ISSN: 0924-1868 (Print) 1573-1391 (Online) (1996)

5. Brusilovsky, P., Millan, P.: User Models for Adaptive Hypermedia y Adaptive Educational Systems. The Adaptive Web, Lecture Notes in Computer Science 4321, Berlin, SpringerVerlag, pp. 3-53. ISSN: 0924-1868 (Print) 1573-1391 (Online) (2007)

6. Praveen, C.: The Eclectic Method- Theory and Its Application to the Learning of English (2013), http://www.ijsrp.org/research-paper-0613/ijsrp-p1844.pdf

7. Aldoobie, N.: ADDIE Model (2015), http://www.aijcrnet.com/journals/Vol_5_No_6_December_2015/10.pdf

8. Brown, H.: Teaching by Principles: An Interactive Approach to Language Pedagogy. Upper Saddle River, New Jersey: Prentice Hall Regents (1994)

9. Weideman, A.: The old and the new: reconsidering eclecticism in language teaching. Unit for Language Skills Development. University of Pretoria. South Africa (2003), http://www.reformationalpublishingproject.com/rpp/docs/6_eclecticism_in_language_07.pdf

10. Amaya, G.: Aprendizaje Autónomo y Competencias. Bogotá (2008)

11. Lara, A.: Aprendizaje Autónomo para estudiantes Universitarios. Colombia: Editorial Universidad de Caldas. Artes y Humanidades (2008)

12. Quesada, C, Sánchez, J.: Calificación y Diagnóstico del Aprendizaje por Computadora. México. Noriega Editores (LIMUSA) (1996)

13. Moreno, A.: Análisis de algunas metodologías para la enseñanza de la Lengua Inglesa en Educación Primaria. Universidad Internacional de la Rioja. Facultad de Educación (2012)

14. Wa'Njogu, K.: Methods of Language Teaching. Yale University (2012)

15. Marcillo, M.A.: La aplicación del Método Ecléctico en el desarrollo de la Expresión Oral del Idioma Inglés. Universidad Central del Ecuador (2017)

16. Aicart, M.: El Método Audio-Lingual. (2015)

17. Rodriguez, M., Rodriguez, J.: Task-Based Language Learning: Old Approach, New Style. A New Lesson to Learn. Vol. 12, No. 2. ISSN 1657-0790. Bogotá, Colombia. Pages 165$178(2010)$ 